\title{
Bi-Level Multi-Objective Stochastic Linear Fractional Programming with General Form of Distribution
}

\author{
Ahmad Yusuf Adhami, Haneefa Kausar* \\ Department of Statistics \& O.R., Aligarh Muslim University, Aligarh, India
}

\begin{abstract}
This paper deals with the stochastic approach of bi-level multi-objective linear fractional programming problem. In this type of bi-level programming problem stochastic nature the right hand side resource vector is considered to follow a general form of distribution $F\left(b_{i}\right)=1-B_{i} e^{-A_{i} h\left(b_{i}\right)}$ [13], which in itself includes many well known distributions such as Pareto distribution, Weibull distribution etc. After converting the problem into an equivalent deterministic form, each level of the problem is transformed into a single objective by using K-T conditions. Finally the problem is solved by Taylors series approach. A numerical example is also presented to illustrate how the proposed approach is utilized.
\end{abstract}

Keywords Bi-level multi-objective programming, Stochastic programming, Fractional programming, Taylor series, KuhnTucker conditions.

AMS 2010 subject classifications 90C15; 90C29; 90C30

DOI: $10.19139 /$ soic.v7i2.373

\section{Introduction}

Bi-level multi-objective programming problem (BLMOPP), which is an apparatus for modeling decentralized decisions, consists of the objectives of the first level decision maker (ULDM) at its first level and that of the objectives of the second level decision maker (LLDM) at the second level.The execution of decision is sequential, from first level to second level; each decision maker (DM) independently controls only a set of decision variables and optimizes the net benefits over a common feasible region. Even though each DM independently tries to optimize their own objective function, the decision may be affected by the actions and reactions of the LLDM due to the dissatisfaction with the decision $[10,16]$.

In other words, although the ULDM independently optimizes its own benefits, the decision may be affected by the reaction of the LLDM. As a result, in most of the practical decision situations, decision deadlock arises frequently and the problem of distribution of proper decision power is encountered. Most of the developments on BLMOP problems focus on bi-level programming problems [6, 23]. There are many algorithms, such as, the K-th best approach [5], Kuhn-Tucker approach, complementarity pivot approach [4], penalty function approach [1, 25], which have been given for solving linear BLP problems. The most popular one is Kuhn-Tucker approach. The fundamental strategy of Kuhn-Tucker is that it replaces the followers problem with its Kuhn-Tucker conditions and appends the resultant system to the leaders problem. The reformulation of the linear BLP problem is a standard mathematical program and relatively easy to solve because all but one constraint is linear. Neglecting or relaxing the constraint leaves a standard linear program that can be solved by using simplex algorithm. Kuhn-Tucker approach has been established to be a valuable analysis tool with a wide range of successful application for linear BLP.

*Correspondence to: Haneefa Kausar (Email: haneefakausar445@gmail.com). Department of Statistics \& O.R., Aligarh Muslim University, Aligarh, UP-202002, India.

ISSN 2310-5070 (online) ISSN 2311-004X (print)

Copyright (C) 2019 International Academic Press 
When the objective function of both level DMs of a BLPP are linear fractional in nature, then BLPP is called bi-level linear fractional programming problem (BLLFPP). Fractional programming problem is the ratio of numerator and denominator. These types of problems have attracted considerable research and interest. Fractional programming is useful in production planning, financial and corporate planning, health care and hospital planning etc . The concept of Multi-objective fractional programming problems and second order generalized hybrid invexity frameworks has given by Verma,[24]. Helmy, et.al[17] have given the idea of stochastic multi-level multi-objective fractional programming problem. Osman, M. S,et al.[21] have also given the concept of stochastic fuzzy multilevel multi-objective fractional programming problem. Kumar, P.et al. [18] have presented the Characterization of Generalized Invexity in multi-objective Fractional Variational Problem. Charles and Dutta [11, 12], have developed a solution procedure for solving multi-objective stochastic fractional programming. Abdelaziz, et al. [3], represented multi-objective programming technique to choose the portfolio best satisfying the decision makers. Most of the probabilistic models assume normal distribution for model coefficients. Sahoo and Biswal [22], have given some deterministic equivalents for the probabilistic problem involving normal and log-normal random variables for joint constraint.

Stochastic linear programming has developed as one of the most important planning tools, with a variety of applications. Stochastic programming deals with situations where some or all the parameters of the optimization problem are described by probabilistic (or random) variables rather than by deterministic one. Maiti, S. K.et.al [19], offered the multi-choice stochastic bi-level programming problem in cooperative nature via fuzzy programming approach. The basic idea in stochastic optimization is to convert the stochastic model into an equivalent deterministic model. A linear or non-linear programming algorithm can be then used to solve the resulting deterministic problem.

Stochastic programming model were first formulated by Dantzig [14], who suggested a two stage programming technique that involves change of stochastic programming model into their equivalent deterministic programming model. This technique suffers from this limitation that it does not allow any constraint to be violated even at specific probability level. This gave rise to the concept of chance constraint programming (CCP), where constraints containing random variables are guaranteed to be satisfied with a certain probability. Chance and Cooper $[8,9]$, originally developed the chance constrained programming technique.

The stochastic bi-level problems with knapsack constraint are that of Ozaltin, et al.[20]. A stochastic version of the bi-level knapsack problem has given by Dempe and Richter in [15]. In their variant of the problem the decision of the leader consists in choosing the (one dimensional) right hand side of the knapsack constraint (i.e. the capacity of the knapsack). The follower has to solve a common knapsack problem that Depends on this value. Ozaltin, et al. extend this model by introducing an uncertainty in the lower level problem. More accurately, they assume that the right hand side of the knapsack constraint in the lower level does not only depend on the leaders decision but also on a random variable.

In this paper, we have considered BLMOSLFPP problem in which there are single ULDM with multi- objectives at the upper level and a single LLDM with multi-objectives at the lower level. The objective functions of the DMs are linear fractional in the nature and the right hand side follows the general form of distributions $F\left(b_{i}\right)=$ $1-B_{i} e^{-A_{i} h\left(b_{i}\right)}$.Its deterministic equivalent form is derived for marginal constraints. Taylor series is used to transform the fractional objective into linear form. Results are also illustrated with the help of numerical example.

\section{SLFP with marginal constraint for general form of distribution}

The mathematical model of a stochastic linear fractional programming problem be expressed as

$$
\max _{k}=\frac{\sum_{j=1}^{n} c_{j}^{k} x_{j}}{\sum_{j=1}^{n} d_{j}^{k} x_{j}} \quad k=1,2 \ldots K
$$

subject to

$$
P\left(\sum_{j=1}^{n} a_{i j} x_{j} \leq b_{i}\right) \geq p_{i}, \quad i=1,2 \ldots n
$$




$$
x_{j} \geq 0, \quad j=1,2 \ldots n
$$

Where $0<p_{i}<1$, and usually close to 1 . Where the decision variable $x_{j}, j=1,2, \ldots n$ and the parameter $a_{i j}, c_{j}$ and $d_{j}$ are deterministic constants and only $b_{i}$ are random variables having a general form of distributions $F\left(b_{i}\right)=$ $1-B_{i} e^{-A_{i} h\left(b_{i}\right)}$. It also given that the $i^{t h}$ random variable $b_{i}$ has two known parameters namely $A_{i}(\neq 0)$, and $B_{i}(>0)$, where $A_{i}$ and $B_{i}$ are such that $F\left(\alpha_{i}\right)=0, F\left(\beta_{i}\right)=1$ and $h\left(b_{i}\right)$ is a monotonic and differentiable function of $b_{i}$ in the interval $\left(\alpha_{i}, \beta_{i}\right)$. In this model, the decision variable $x_{j}, j=1,2, \ldots n$, are treated as deterministic decision variables.

The probability density function of the random variable $b_{i}$ is given by

$$
f\left(b_{i}\right)=A_{i} B_{i} e^{-A_{i} h\left(b_{i}\right)} h^{\prime}\left(b_{i}\right)
$$

Equation (2)can be expressed as

$$
P\left(b_{i} \geq y_{i}\right) \geq p_{i}, i=1,2 \ldots m
$$

where

$$
y_{i}=\sum_{j=1}^{n} a_{i j} x_{j}
$$

Equation (5) can be restated as

$$
\int_{y_{i}}^{\beta_{i}} A_{i} B_{i} e^{-A_{i} h\left(b_{i}\right)} h^{\prime}\left(b_{i}\right) d b_{i} \geq p_{i}, \quad i=1,2 \ldots m
$$

After integration,we have

$$
B_{i} e^{-A_{i} h\left(y_{i}\right)} \geq p_{i} \text { as } B_{i} e^{-A_{i} h\left(\beta_{i}\right)}=0
$$

\subsection{Few special classes of distributions}

(1) When $b_{i}{ }^{\prime} \mathbf{s}$ follow Weibull distribution

$$
F\left(b_{i}\right)=1-e^{-\theta_{i} b_{i} a_{i}}, 0 \leq b_{i}<\infty, a_{i}>0, \theta_{i}>0
$$

Here $A_{i}=\theta_{i}, B_{i}=1, h\left(b_{i}\right)=b_{i}{ }^{a_{i}}$ Now from equation (6), we

$$
e^{-\theta_{i} y_{i}^{a_{i}}} \geq p_{i}
$$

This can be simplified as

$$
y_{i} \leq\left[-\frac{1}{\theta}{ }_{i} \log \left(p_{i}\right)\right]^{1 / a_{i}}
$$

So, the deterministic mathematical SLFP model can be expressed as

$$
\begin{aligned}
& \max _{k}=\frac{\sum_{j=1}^{n} c_{j}^{k} x_{j}}{\sum_{j=1}^{n} d_{j}^{k} x_{j}} \quad k=1,2 \ldots K \\
& \text { subject to }
\end{aligned}
$$

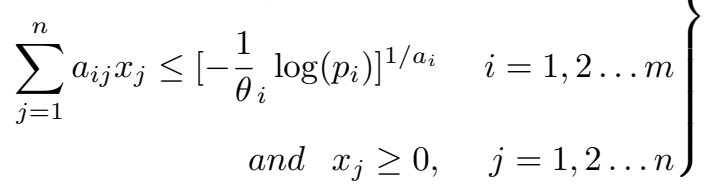


Note:we can also take $A_{i}=1, B_{i}=1, h\left(b_{i}\right)=\theta_{i} b_{i}{ }^{a_{i}}$ for the Weibull distribution.

(2) When $b_{i}{ }^{\prime} \mathbf{s}$ follow Burr Type XII distribution

The distribution function of Burr type XII distribution is given by

$$
F\left(b_{i}\right)=1-\left(1+\theta_{i} b_{i i}^{a}\right)^{-} \lambda_{i}, 0 \leq b_{i}<\infty, a_{i}>0, \theta_{i}>0, \lambda_{i}>0
$$

Here $A_{i}=\lambda_{i}, B_{i}=1, h\left(b_{i}\right)=\log \left(1+\theta b_{i}{ }^{a_{i}}\right)$

Now from equation (6), we have

$$
e^{-\lambda_{i} \log \left(1+\theta_{i} y_{i i}^{a}\right) \geq p_{i}}
$$

This can be simplified as

$$
y_{i} \leq\left[\frac{p_{i}^{-1 / \lambda_{i}}-1}{\theta}\right]^{1 / a_{i}}
$$

So,the deterministic mathematical model of SLFP can be expressed as

$$
\begin{aligned}
& \left.\max _{k}=\frac{\sum_{j=1}^{n} c_{j}^{k} x_{j}}{\sum_{j=1}^{n} d_{j}^{k} x_{j}} \quad k=1,2 \ldots K\right) \\
& \text { subject to } \\
& \left.\begin{array}{rl}
\sum_{j=1}^{n} a_{i j} x_{j} \leq\left[\frac{p_{i}{ }^{-1 / \lambda_{i}}-1}{\theta}\right]_{i}^{1 / a_{i}} & i=1,2 \ldots m \\
\text { and } x_{j} \geq 0, \quad j=1,2 \ldots n
\end{array}\right\}
\end{aligned}
$$

Note: we can also take $A_{i}=\lambda_{i}, B_{i}=1, h\left(b_{i}\right)=\log \left(1+\theta b_{i}{ }^{a_{i}}\right)$ for the Burr Type XII distribution.

(3) When $\mathbf{b}_{\mathbf{i}}{ }^{\prime} \mathbf{s}$ follow Beta distribution of first kind

The distribution function of Beta distribution of first kind is given by

$$
F\left(b_{i}\right)=1-\left(\frac{\lambda_{i}-b_{i}}{\lambda_{i}-\delta_{i}}\right)^{a_{i}}, 0<\delta_{i} \leq b_{i} \leq \lambda_{i}, a_{i}
$$

Here $A_{i}=\lambda_{i}, B_{i}=1, h\left(b_{i}\right)=\log \left(\frac{\lambda_{i}-b_{i}}{\lambda_{i}-\delta_{i}}\right)^{a_{i}}$

Now from equation(6), we have $e^{a_{i} \log \left(\frac{\lambda_{i}-b_{i}}{\lambda_{i}-\delta_{i}}\right)} \geq p_{i}$

This can be simplified as

$$
\left.\begin{array}{c}
y_{i} \leq \lambda_{i}\left(1-p_{i}{ }^{1} / a_{i}\right)+p_{i}{ }^{1} / a_{i} \delta_{i} \\
\max _{k}=\frac{\sum_{j=1}^{n} c_{j}^{k} x_{j}}{\sum_{j=1}^{n} d_{j}^{k} x_{j}} \quad k=1,2 \ldots K \\
\text { subject to } \\
\sum_{j=1}^{n} a_{i j} x_{j} \leq \lambda_{i}\left(1-p_{i}{ }^{1} / a_{i}\right)+p_{i}{ }^{1} / a_{i} \delta_{i} \\
x_{j} \geq 0, \quad i=1,2 \ldots m \\
j=1,2 \ldots n
\end{array}\right\}
$$


Note: we can also take $A_{i}=\lambda_{i}, B_{i}=1, h\left(b_{i}\right)=a_{i} \log \left(\frac{\lambda_{i}-b_{i}}{\lambda_{i}-\delta_{i}}\right)$ for the Beta distribution of First kind.

(4) When $b_{i}{ }^{\prime} s$ follow Pareto distribution

The distribution function of Pareto distribution is given by

$$
F\left(b_{i}\right)=1-\lambda_{i}^{a_{i}} b_{i}^{-a_{i}}, 0<\lambda_{i} \leq b_{i} \infty, a_{i}>0
$$

Here $A_{i}=a_{i}, B_{i}=\lambda_{i i}{ }^{a}, h\left(b_{i}\right)=\log \left(b_{i}\right)$

Now from equation (6), we have

$$
\lambda_{i i}^{a} e^{-a_{i} \log \left(y_{i}\right)} \geq p_{i}
$$

This can be simplified as

$$
y_{i} \leq \frac{\lambda_{i}}{p_{i}{ }^{1 / a_{i}}}
$$

So, the deterministic mathematical SLFP model can be expresses as

$$
\begin{aligned}
& \max z_{k}=\frac{\sum_{j=1}^{n} c_{j}^{k} x_{j}}{\sum_{j=1}^{n} d_{j}^{k} x_{j}} \quad k=1,2 \ldots K \\
& \text { subject to } \\
& \sum_{j=1}^{n} a_{i j} x_{j} \leq \frac{\lambda_{i}}{p_{i}^{1 / a_{i}}} \quad i=1,2 \ldots m \\
& \text { and } x_{j} \geq 0, \quad j=1,2 \ldots n \text { ) }
\end{aligned}
$$

Note: we can also take $A_{i}=1, B_{i}=\lambda_{i}^{{ }^{a}}, h\left(b_{i}\right)=a_{i} \log \left(b_{i}\right)$ for the Pareto distribution.

(5) When $b_{i}{ }^{\prime} \mathbf{s}$ follow Power function distribution

The distribution function of Power Function distribution is given by

$$
F\left(b_{i}\right)=\lambda_{i}^{a_{i}} b_{i}^{-a_{i}}, 0 \leq b_{i} \leq \lambda_{i}, a_{i}>0, \lambda_{i}>0
$$

Here $A_{i}=-1, B_{i}=1, h\left(b_{i}\right)=\log \left(1-\lambda_{i}^{-a_{i}} b_{i}^{-a_{i}}\right)$ Now from equation(6), we have

$$
e^{\log \left(1-\lambda_{i}^{-a_{i}} y_{i}^{a_{i}}\right)} \leq p_{i}
$$

This can be simplified as

$$
y_{i} \leq \lambda_{i}\left(1-p_{i}\right)^{1 / a_{i}}
$$

So,the deterministic mathematical model of SLFP can be expressed as

$$
\begin{aligned}
& \max z_{k}=\frac{\sum_{j=1}^{n} c_{j}^{k} x_{j}}{\sum_{j=1}^{n} d_{j}^{k} x_{j}} \quad k=1,2 \ldots K \\
& \text { subject to } \\
& \sum_{j=1}^{n} a_{i j} x_{j} \leq \lambda_{i}\left(1-p_{i}\right)^{1 / a_{i}} \quad i=1,2 \ldots m \\
& \text { and } x_{j} \geq 0, \quad j=1,2 \ldots n
\end{aligned}
$$




\section{Problem formulation}

Suppose that there are two levels in a hierarchy structure with upper level decision maker (ULDM) and lower level decision maker (LLDM). Let the vector of decision variables $x=\left(x_{1}, x_{2}, x_{3}\right) \in R^{n_{1}}$ be partitioned between the two planners. The upper level decision maker has control over the vector $x_{1} \in R^{n_{2}}, x_{3} \in R^{n_{3}}$ where $n=$ $n_{1}+n_{2}+n_{3}$ Now further we assume that

$$
F_{i}\left(x_{1}, x_{2}, x_{3}\right): R^{n_{1}} \times R^{n_{2}} \times R^{n_{3}} \rightarrow R^{m_{i}}, i=1,2,3
$$

The upper level and lower level vector objective function respectively.

So the BLMO-SLFP problem of maximization type may be formulated as follows [2, 7]:

[ULDM]

$$
\max _{x_{1}} F_{1}\left(x_{1}, x_{2}, x_{3}\right)=\operatorname{Max}\left(f_{11}\left(x_{1}, x_{2}, x_{3}\right), f_{12}\left(x_{1}, x_{2}, x_{3}\right) \ldots \ldots f_{1} m_{1}\left(x_{1}, x_{2}, x_{3}\right)\right)
$$

Where $x_{2}, x_{3} \ldots \ldots . x_{n}$ solves

[LLDM]

$$
\begin{aligned}
& \max _{x_{2}} F_{2}\left(x_{1}, x_{2}, x_{3}\right)=\operatorname{Max}\left(f_{21}\left(x_{1}, x_{2}, x_{3}\right), f_{22}\left(x_{1}, x_{2}, x_{3}\right) \ldots \ldots f_{2} m_{2}\left(x_{1}, x_{2}, x_{3}\right)\right) \\
& \max _{x_{3}} F_{3}\left(x_{1}, x_{2}, x_{3}\right)=\operatorname{Max}\left(f_{31}\left(x_{1}, x_{2}, x_{3}\right), f_{32}\left(x_{1}, x_{2}, x_{3}\right) \ldots \ldots f_{3} m_{3}\left(x_{1}, x_{2}, x_{3}\right)\right) \\
& \max _{x_{n}} F_{n}\left(x_{1}, x_{2}, x_{3}\right)=\operatorname{Max}\left(f_{n 1}\left(x_{1}, x_{2}, x_{3}\right), f_{n 2}\left(x_{1}, x_{2}, x_{3}\right) \ldots \ldots f_{n} m_{n}\left(x_{1}, x_{2}, x_{3}\right)\right)
\end{aligned}
$$

subject to

$$
P\left[\sum_{j=1}^{n} a_{i j} x_{j} \leq b_{1}, \sum_{j=1}^{n} a_{i j} x_{j} \leq b_{2} \ldots \ldots, \sum_{j=1}^{n} a_{i j} x_{j} \leq b_{m}\right] \geq p
$$

Where

$$
f_{i j}\left(x_{1}, x_{2}, x_{3}\right)=\frac{c_{j}^{k} x_{j}+\gamma_{i j}}{d_{j}^{k} x_{j}+\theta_{i j}}
$$

for $\mathrm{i}=1$, we have $j=1,2, \ldots, m_{1}$, for ULDM objective functions, for $\mathrm{i}=1,2,3 \ldots$. p,we have $j=1,2, \ldots ., m_{i}$, for LLDM objective functions, where $m_{i}, i=1,2, \ldots, p$ is the number of decision makers LLDM objective function, $\mathrm{m}$ is the number of constraint $A_{i}$ is the coefficient of matrices of sizen $m \times n_{i}, c_{j}^{k}, d_{j}^{k} \in R^{n}, d_{j}^{k} x_{j}+\theta_{i j}>0$ for all $x \in G$ and $\gamma_{i j}, \theta_{i j}$ are constants (for $i=1,2 \ldots, p \& j=1,2, \ldots, m_{i}$ )

\section{The Taylor's series and Kuhn-Tucker conditions for solving BLMOS-LFPP}

In the deterministic Bi-level fractional programming we can transform objective functions by using 1st order Taylor series into Bi-level linear programming problem for the ULDM and LLDM. This approach can be explained in four steps.

Step1:Determine $x_{i j}^{*}=\left(x_{i j}^{1} *, x_{i j}^{2} *, \ldots . x_{i} j^{p} *\right)\left(i=1,2 \ldots . . p j=1,2, \ldots . . m_{i}\right)$ which is the values that is used to 
maximize the each of the objective functions in upper level and lower level $F_{i j}(x)\left(i=1,2 \ldots . . p j=1,2, \ldots . . m_{i}\right)$ where $m$ is the number of the variables.

Step2: Transform the objective functions $F_{i j}(x)\left(i=1,2 \ldots . . p j=1,2, \ldots . . m_{i}\right)$ by using the first-order Taylor polynomial series.

$F_{i j}(x)=F_{i j}\left(x_{i j}^{*}\right)=F_{i j}\left(x_{i j}^{*}\right)+\sum_{k=1}^{p}\left(x_{k}-x_{i j}^{k} *\right)$

Step3: Sum the objective functions together for the upper level. Note that problem is solved by assuming that the objective functions in upper level are equal

$$
P(x)=\sum_{j=2}^{m_{1}}\left(F_{1 j}\left(x_{1 j}^{*}\right)+\sum_{k=1}^{p}\left(x_{k}-x_{1 j}^{k} *\right) \frac{\partial F_{1 j}\left(x_{1 j}^{*}\right)}{\partial x_{k}}\right.
$$

Step4: After applying the Kuhn-Tucker conditions to the lower level of the objective problem, we find satisfactory $x^{*}=\left(x_{1}^{*}, x_{2}^{*}, x_{3}^{*}, x_{p}^{*}\right)$ by solving the reduced problem to a single objective.

BL-SLFPP is converted into a new mathematical model. This model is represented as follows:

$\operatorname{Max} P(x)$

s.t

$A_{1} x_{1}+A_{2} x_{2}+\ldots+A_{p} x_{p}+u=b$

$$
w A_{i}-v_{i}=\sum_{j=2}^{m_{i}} \frac{\partial F_{i j}\left(x_{i j}^{*}\right)}{\partial x_{i}}
$$

$w u=0, x_{i} v_{i}=0$

$x_{i}, w, u, v_{i}>=0 \quad i=2,3, \ldots, p$

In this method, a zero-one variable, $\eta$ and $\xi_{i}$ is added for each constraint $w u=0$ and $x_{i} v_{i}=0$ respectively. In addition, each of these constraints is replaced by two linear inequalities involving $\eta$ and $\xi_{i}$ and M,a large positive. The auxiliary formulation now becomes

$\operatorname{MaxP}(\mathrm{x})$

s.t

$A_{1} x_{1}+A_{2} x_{2}+\ldots+A_{p} x_{p}+u=b$

$$
\left.\left.w A_{i}-v_{i}=\sum_{(} j=2\right)^{(} m_{i}\right) \partial F_{i} j\left(x_{i} j^{*}\right) \partial x_{i}
$$

$w \leq M \eta, u \leq M(1-\eta)$

$x_{i} \leq M x i_{i}, v \leq M\left(1-x i_{i}\right)$

$\eta, x i_{i} \in 0,1$

$x_{i}, w, u, v_{i} \geq 0 \quad i=1,2,3, \ldots, p$

\section{An illustrative numerical example}

$[\mathrm{ULDM}] \max _{x_{1}}\left(F_{11}=\frac{10 x_{1}+6 x_{2}+3 x_{3}}{x_{1}+2 x_{2}+x_{3}+1}, F_{12}=\frac{6 x_{1}+8 x_{2}+10 x_{3}}{x_{1}+x_{2}+2 x_{3}+1}\right)$

Where $x_{2}, x_{3}$ solve

$[\mathrm{LLDM}] \max _{x_{2}}\left(F_{21}=\frac{4 x_{1}+6 x_{2}+9 x_{3}}{2 x_{1}+x_{2}+x_{3}+1}, F_{22}=\frac{7 x_{1}+9 x_{2}+5 x_{3}}{x_{1}+2 x_{2}+x_{3}+1}\right)$

$\max _{x_{3}}\left(F_{31}=\frac{6 x_{1}+8 x_{2}+4 x_{3}}{2 x_{1}+x_{2}+x_{3}+1}, F_{33}=\frac{10 x_{1}+6 x_{2}+3 x_{3}}{2 x_{1}+x_{2}+x_{3}+1}\right)$

Subject to

$$
P\left(x_{1}+x_{2}+x_{3} \leq b_{1}\right) \geq .90
$$




$$
\begin{gathered}
P\left(3 x_{1}+2 x_{2}+x_{3} \leq b_{2}\right) \geq .98 \\
P\left(x_{1}+2 x_{2}+3 x_{3} \leq b_{3}\right) \geq .95 \\
P\left(2 x_{1}+x_{2}+x_{3} \leq b_{4}\right) \geq .90 \\
x_{1}, x_{2}, x_{3} \geq 0
\end{gathered}
$$

where $b_{1}$ follows Power Function distribution with parameter $\lambda=10, a=5 ; b_{2}$ follows Pareto distribution with parameter $\lambda=8, a=2 ; b_{3}$ follows Beta of first kind distribution with parameterlambda $=15, a=10, \delta=3 ; b_{4}$ follows Weibull distribution with parameter $\theta=1 / 5, a=10 ; b_{5}$ follows Burr type XII distribution with parameter $\lambda=1 / 10, \theta=1 / 5, a=1 / 5$.

Using the well known concept of weighting characteristic of the objective functions and from equations (9),(12),(15),(18) and (21) the deterministic model of the above MOSLP problem is.

[ULDM]

$$
\operatorname{Max}\left(F_{11}=\frac{10 x_{1}+6 x_{2}+3 x_{3}}{x_{1}+2 x_{2}+x_{3}+1}, F_{12}=\frac{6 x_{1}+8 x_{2}+10 x_{3}}{x_{1}+x_{2}+2 x_{3}+1}\right)
$$

Where $x_{2}, x_{3}$ solve

[LLDM]

$$
\begin{gathered}
\operatorname{Max}\left(F_{21}=\frac{4 x_{1}+6 x_{2}+9 x_{3}}{2 x_{1}+x_{2}+x_{3}+1}, F_{2} 2=\frac{7 x_{1}+9 x_{2}+5 x_{3}}{x_{1}+2 x_{2}+x_{3}+1}\right) \\
\operatorname{Max}\left(F_{31}=\frac{6 x_{1}+8 x_{2}+4 x_{3}}{2 x_{1}+x_{2}+x_{3}+1}, F_{3}=\frac{10 x_{1}+6 x_{2}+3 x_{3}}{2 x_{1}+x_{2}+x_{3}+1}\right)
\end{gathered}
$$

Subject to

$$
\begin{gathered}
x_{1}+x_{2}+x_{3} \leq 6 \\
3 x_{1}+2 x_{2}+x_{3} \leq 8 \\
x_{1}+2 x_{2}+3 x_{3} \leq 3 \\
2 x_{1}+x_{2}+x_{3} \leq 9 \\
10 x_{1}+x_{2}+x_{3} \leq 10 \\
x_{1}, x_{2}, x_{3} \geq 0
\end{gathered}
$$

If the problem is solved for each objective functions, one by one then $f_{11}(1,0,0), f_{12}(0,0,1), f_{21}(2.67,0,0), f_{22}(0,1.5,0), f_{31}(0,1.5,0)$ and $f_{32}(2.50,0.25,0)$ are obtained. Now the objective functions are transformed by using first-order Taylor polynomial series. 


$$
\begin{gathered}
F_{11} \cong \bar{F}_{11}(x)=F_{11}(1,0,0)+\left(x_{1}-1\right) \times \frac{\partial}{\partial x_{1}}+\left(x_{2}-0\right) \times \frac{\partial}{\partial x_{2}}+\left(x_{3}-0\right) \times \frac{\partial}{\partial x_{3}} F_{11}(1,0,0) \\
F_{11}=2.5+2.5 x_{1}-2 x_{2}-x_{3}
\end{gathered}
$$

Similarly, the other objective functions are transformed on using first-order Taylor polynomial series as follows:

$$
\begin{aligned}
& F_{12}(x)=2.88+4.8 x_{1}+1.28 x_{2}+0.16 x_{3} \\
& F_{21}(x)=2.25-2.5 x_{1}+0.75 x_{2}+2.25 x_{3} \\
& F_{22}(x)=3.7+0.52 x_{1}-0.32 x_{2}-0.03 x_{3} \\
& F_{31}(x)=3.36-1.44 x_{1}+1.28 x_{2}-0.32 x_{3} \\
& F_{32}(x)=3.57+0.24 x_{1}+0.28 x_{2}-0.19 x_{3}
\end{aligned}
$$

The $\mathrm{P}(\mathrm{x})$ is obtained by adding (32) and (33) as follows:

$$
P(x)=F_{11}(x)+F_{1} 2(x)=5.38+7.3 x_{1}-0.72 x_{2}-0.84 x_{3}
$$

After applying the Kuhn-Tucker conditions to the lower level of the objectives problem, a new auxiliary problem is to be solved

$$
\begin{aligned}
& \operatorname{Max} P(x)=5.38+7.3 x_{1}-0.72 x_{2}-0.84 x_{3} \text { S.t } \\
& x_{1}+x_{2}+x_{3}+u_{1}=6 \\
& 3 x_{1}+2 x_{2}+x_{3}+u_{2}=8 \\
& x_{1}+2 x_{2}+3 x_{3}+u_{3}=3 \\
& 2 x_{1}+x_{2}+x_{3}+u_{4}=9
\end{aligned}
$$

$$
10 x_{1}+x_{2}+x_{3}+u_{5}=10
$$

$w_{1}+2 w_{2}+2 w_{3}+w_{4}+w_{5}-v_{1}=0.75-0.32$

$w_{1}+w_{2}+3 w_{3}+w_{4}+w_{5}-v_{2}=-0.19-0.84$

$w_{j} \leq M \eta_{j}, u_{j} \leq\left(1-\eta_{j}\right), x_{i} \leq M \xi_{i}, v_{i} \leq M\left(1-\xi_{i}\right), \eta_{j}, \xi_{i} \in[0,1]$

$x_{1}, x_{2}, w_{j}, u_{j}, v_{i} \geq 0, i=1,2 j=1,25$

We solve the problem for $\mathrm{M}=1000$ and the solution is obtained as follow

$x_{1}^{*}=1, x_{2}^{*}=0, x_{3}^{*}=0$

$F_{11}=5, F_{12}=3, F_{21}=1.33, F_{22}=3.5, F_{31}=2, F_{32}=3.33$

\section{Conclusion}

In this paper, we developed a stochastic programming model, which consider a ratio of two linear functions in the multi-objectives and probabilistic constraints. The main contribution of this paper is to solve BLMO-SLFPP by converting it into deterministic form where the right hand side resource vector follows a general form of distribution which includes distributions like Power function Distribution, Pareto Distribution etc. 


\section{Acknowledgement}

The first author gratefully acknowledges the financial support from UGC-New Delhi. (UGC-BSR start up grant no.F30-62/2014(BSR)).

\section{REFERENCES}

1. Aiyoshi E., and Shimizu, K. (1981), Hierarchical decentralized systems and its new solution by a barrier method, IEEE Transactions on Systems, Man and Cybernetics, (6), 444-449.

2. Abo-Sinna, M. A. (2001), A bi-level non-linear multi-objective decision making under fuzziness, Opsearch New Delhi, 38(5) (2001) 484-495.

3. Abdelaziz, F. B., Aouni, B., and El Fayedh, R. (2007), Multi-objective stochastic programming for portfolio selection, European Journal of Operational Research, 177(3), 1811-1823.

4. Bialas, W., Karwan, M., and Shaw, J. (1980), A parametric complementary pivot approach for two-level linear programming, State University of New York at Buffalo, 57.

5. Bialas, W. F., and Karwan, M. H. (1984), Two-level linear programming, Management science, 30(8), 1004-1020.

6. Ben-Ayed, O. (1993), Bilevel linear programming, Computers and operations research, 20(5), 485-501.

7. Baky, I. A. (2009), Fuzzy goal programming algorithm for solving decentralized bi-level multi-objective programming problems, Fuzzy sets and systems, 160(18), 2701-2713.

8. Charnes, A., and Cooper, W. W. (1959), Chance-constrained programming, Management science, 6(1), 73-79.

9. Charnes, A., and Cooper, W. W. (1963), Deterministic equivalents for optimizing and satisficing under chance constraints, Operations research, 11(1), 18-39.

10. Candler, W., and Townsley, R. (1982), A linear two-level programming problem, Computers \& Operations Research, 9(1), 59-76.

11. Charles V., and Dutta, D. (2003), Bi-weighted multi-objective stochastic fractional programming problem with mixed constraints, In Proceedings of the 2nd National Conference on Mathematical and Computational Methods (pp. 29-36). New Delhi, India: Allied.

12. Charles, V., and Dutta, D. (2006), Extremization of multi-objective stochastic fractional programming problem, Annals of Operations Research, 143(1), 297-304.

13. Charles, V., Ansari, S. I., and Khalid, M. M. (2011), Multi-objective stochastic linear programming with general form of distributions, Int J Oper Res Optim, 2(2), 261-278.

14. Dantzig, G. B.(1955), Linear programming under uncertainty, Management science, 1(3-4) 197-206.

15. Dempe, S., and Richter, K. (2000), Bilevel programming with knapsack constraints, TU Bergakademie, Fakult?t fr Mathematik und Informatik.

16. Fortuny-Amat, J., and McCarl, B. (1981), A representation and economic interpretation of a two-level programming problem, Journal of the operational Research Society, 783-792.

17. Helmy, Y. M., Emam, O. E., and Abdelwahab, A. M. (2015), On stochastic multi-level multi-objective fractional programming problems, Journal of Statistics Applications and Probability, 4(1), 93

18. Kumar, P., Dagar, J., and Sharma, B. (2016), Characterization of Generalized Invexity in Multi-objective Fractional Variational Problem, Statistics, Optimization and Information Computing, 4(4), 342-349.

19. Maiti, S. K., and Roy, S. K. (2016), Multi-choice stochastic bi-level programming problem in cooperative nature via fuzzy programming approach, Journal of Industrial Engineering International, 12(3), 287-298.

20. Ozaltn, O. Y., Prokopyev, O. A., and Schaefer, A. J. (2010), The bilevel knapsack problem with stochastic right-hand sides, Operations Research Letters, 38(4), 328-333.

21. Osman, M. S., Emam, O. E., and El Sayed, M. A. (2017), Stochastic fuzzy multi-level multi-objective fractional programming problem, A FGP approach. OPSEARCH, 54(4), 816-840

22. Sahoo, N. P., and Biswal, M. P. (2005), Computation of Probabilistic linear programming problems involving normal and log-normal random variables with a joint constraint, Computer Mathematics, 82(11), 1323-1338.

23. Saraj, M.,\& Safaei, N. (2012), Solving bi-level programming problems on using global criterion method with an interval approach, Applied Mathematical Science, 6(23), 1135-1141.

24. Verma, R. (2014), Multi-objective fractional programming problems and second order generalized hybrid invexity frameworks, Statistics, Optimization and Information Computing, 2(4), 280-304

25. White, D. J., and Anandalingam, G. (1993), A penalty function approach for solving bi-level linear programs, Journal of Global Optimization, 3(4), 397-419. 